\title{
Origin and Evolution of the Moon
}

\section{Cuixiang Zhong}

Department of science and technology,Jiangxi Normal University, Nanchang 330022, China

email: cuixiang_zhong@ sohu.com

\begin{abstract}
Since the Moon is the only natural satellite of the Earth, the research on the formation of the Moon can not only find out the formation mechanism of the satellites of Solar System planets but also reveal the evolution law of galaxies in the universe. Hence many hypotheses have been proposed for the Moon's formation, including fission,capture,condensation, and impact event hypothesis, but they all have problems. Recently, the author of this abstract discovered the formation mechanism of the Moon, which can be called "evolution theory", and described as follows: During some violent volcanic eruptions of the Earth, some rock debris such as pumice through deep rock hole could achieve a velocity no less than the first cosmic velocity $(7.9 \mathrm{~km} / \mathrm{s})$ to enter an orbit around the Earth, one of the biggest debris is the young Moon.

The orbit of the young Moon might be much closer to the Earth than it is today. There were a lot of ejecta from the Earth in the space. Hence, the Moon has merged these ejecta to become larger and larger, and farther and farther away from the Earth.This can be proved as follows: When the Moon moved around the Earth normally, the centrifugal force produced by the Moon's rotation around the Earth and the Earth's gravitation pull on the Moon had the same size. Let $M$ be the mass of the Earth, $m_{1}$ be the mass of the Moon, $r_{m}$ be the radius of the Moon, $r$ be the centroid distance between the Earth and the Moon, $v$ be the tangential velocity of the Moon around the Earth, then $G m_{1} M / r^{2}=m_{1} v^{2} / r$, therefore $v=\sqrt{G M / r}$. Near the orbit of the Moon, there were also many smaller prograde planetesimals moving around the Earth in circular orbits of radius $r_{x}\left(r-r_{m}<r_{x}<r\right)$ with velocity $v_{x}$. Since $v_{x}=\sqrt{G M / r_{x}}>\sqrt{G M / r}$, which implies $v_{x}>v$, these planetesimals would finally catch and merge with the Moon.Especially,if a planetesimals was large enough, it would impact the Moon forcefully, making the Moon's velocity increase to a larger value $v_{2}$. Then $m_{1} v_{2}^{2} / r>m_{1} v^{2} / r=G m_{1} M / r^{2}$, therefore the Moon moved a reasonable distance away from the Earth.

The early orbit of the Moon was much closer to the Earth's atmosphere than it is now, so the Moon had absorbed much water vapor from the atmosphere to form lunar maria. Due to the constant erosion of water,a series of volcanic eruptions occurred at the bottom of lunar maria.Violent eruption could also change the orbit of the Moon around the Earth. Since most maria are on the near-side visible from Earth, so many violent volcanic eruptions at the bottom of these lunar maria could push the moon away from the Earth. This can be proved as follows: Let $M$ be the mass of the Earth, $m_{1}$ be the mass of the Moon left by the volcanic eruption, $m_{2}$ be the total mass of erupted matter, $m_{0}\left(=m_{1}+m_{2}\right)$ be the total mass of the Moon before the eruption, $v$ be the tangential velocity of the Moon around the Earth, $r_{0}$ and $r_{1}$ be the centroid distance between the Earth and the Moon before or after the eruption respectively; since the volcano ejected many substances toward the Earth, $r_{1}>r_{0}$. Before the eruption, the centrifugal force produced by the Moon's rotation around the Earth and the Earth's gravitation pull on the Moon had the same size, therefore $G m_{0} M / r_{0}{ }^{2}=m_{0} v^{2} / r_{0}$, which implies $G M / r_{0}{ }^{2}=v^{2} / r_{0}$. After the eruption, if the Moon hadn't changed its orbit then its velocity around the Earth was still $v$,so $G m_{1} M / r_{1}{ }^{2}=\left(G m_{1} M / r_{0}{ }^{2}\right)\left(r_{0}{ }^{2} / r_{1}{ }^{2}\right)=\left(m_{1} v^{2} / r_{0}\right)\left(r_{0}{ }^{2} / r_{1}{ }^{2}\right)=\left(m_{1} v^{2} / r_{1}\right)\left(r_{0} / r_{1}\right)<\left(m_{1} v^{2} / r_{1}\right)$, adding the continuous giant reaction force produced by volcanic eruption, the join force could push the Moon away from the Earth, even to an orbit where the water vapor evaporated from the Moon would be attracted back to the Earth,leaving dry lunar maria.
\end{abstract}

Keywords. Moon, origin, evolution, Earth, volcanic eruption, the Galaxy 